\title{
Stability of Spheromaks Compressed by Liquid Walls
}

\author{
T. K. Fowler
}

August 17, 1999

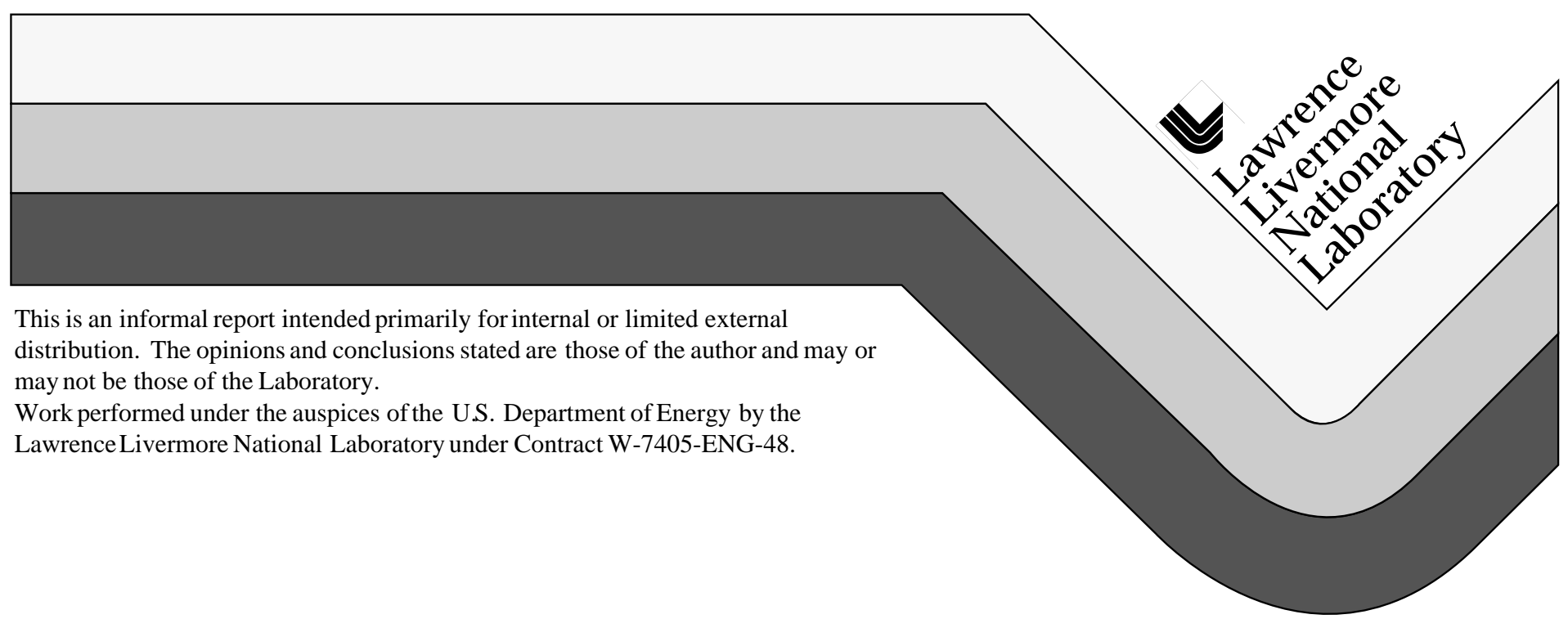




\section{DISCLAIMER}

This document was prepared as an account of work sponsored by an agency of the United States Government. Neither the United States Government nor the University of California nor any of their employees, makes any warranty, express or implied, or assumes any legal liability or responsibility for the accuracy, completeness, or usefulness of any information, apparatus, product, or process disclosed, or represents that its use would not infringe privately owned rights. Reference herein to any specific commercial product, process, or service by trade name, trademark, manufacturer, or otherwise, does not necessarily constitute or imply its endorsement, recommendation, or favoring by the United States Government or the University of California. The views and opinions of authors expressed herein do not necessarily state or reflect those of the United States Government or the University of California, and shall not be used for advertising or product endorsement purposes.

This report has been reproduced directly from the best available copy.

Available to DOE and DOE contractors from the Office of Scientific and Technical Information P.O. Box 62, Oak Ridge, TN 37831

Prices available from (423) 576-8401

Available to the public from the National Technical Information Service

U.S. Department of Commerce 5285 Port Royal Rd. Springfield, VA 22161 


\title{
STABILITY OF SPHEROMAKS COMPRESSED BY LIQUID WALLS
}

\author{
T. K. Fowler
}

August 17, 1999

\begin{abstract}
We consider Rayleigh-Taylor instability of the liquid-plasma interface of a spheromak ignited by slow compression discussed previously. We conclude that instability may indeed occur despite the stabilizing influence of magnetic shear in the spheromak. If it occurs, instability would be greatest for modes concentrated toward the midplane. As for the cylindrical LINUS configuration, rotation about the geometric axis would stabilize these modes, but at the price of roughly doubling the input energy and reducing the gain. However, even in the absence of rotation, in a sphere instability occurs only at the end of compression near the stagnation point. Revised estimates of the fusion energy gain taking this brief period of instability into account still give, within the uncertainties, a gain $\mathrm{G} \approx 20$ for our earlier example with an input energy of $150 \mathrm{MJ}$ and fusion yield of $3 \mathrm{GJ}$.
\end{abstract}

$$
* * * * * * * * *
$$

\section{Formulation of the Stability Problem}

We consider a spherical shell of conducting liquid imploding on a spheromak inside the shell, discussed in Reference [1]. As before, we assume incompressibility and only later correct for the energy of compression in the liquid. Ignoring viscosity, both the liquid and the spheromak are described by the MHD magnetic equation and the momentum equation given by:

$$
\rho \mathrm{d} \mathbf{v} / \mathrm{dt} \quad=-\nabla \mathrm{P}+\mathbf{C}
$$

where $\mathrm{P}=\mathrm{p}+\mathrm{B}^{2} / 2 \mu_{\mathrm{o}}$ with pressure $\mathrm{p}$ and $\mathbf{C}$ is the magnetic curvature term. Linearizing gives a force term $\rho_{1} \mathbf{g}$ where [2]:

$$
\text { g } \quad=\quad-\mathrm{d} \mathbf{v}_{\mathrm{o}} / \mathrm{dt}
$$

The effective gravity $\mathbf{g}$, not present for a static non-accelerating equilibrium state, is a potential source of Rayleigh-Taylor instability at the interface where $\rho_{1}=-\xi . \nabla \rho_{\mathrm{o}}$ is 
large. Neglecting curvature and $\mathbf{v . \nabla}$ in the convective derivative gives the standard equation for purely growing or oscillating Rayleigh-Taylor modes [3]:

$$
\xi^{\prime \prime}+\left(\rho^{\prime} / \rho\right) \xi^{\prime}-k^{2}\left(1-g\left(\rho^{\prime} / \rho\right) \omega^{-2}\right) \xi=0
$$

where $\xi$ is the displacement perpendicular to the surface, (') denotes derivatives perpendicular to the surface, $\mathrm{k}$ lies in the surface and $\mathrm{g}$ is the effective gravity due to acceleration. The steep gradient causes the solution to localize near the surface of the liquid with frequency:

$$
\omega=(\mathrm{kg})^{1 / 2} \quad \text { for } \mathrm{k}<\left(\rho^{\prime} / \rho\right)
$$

Hence modes grow if $\mathrm{g}<0$ (gravity pointing inward toward the spheromak). As noted below, $g$ at the surface changes sign during compression, so that instability, if it occurs, does so near the end of the compression cycle when the compression of the field begins to decelerate the liquid.

\section{Calculation of $\mathbf{g}$}

The effective gravity g can be calculated by integrating Eq. (1) over $\mathrm{r}$ and using incompressibility to determine $\mathrm{v}(\mathrm{r})$ from $\nabla . \mathbf{v}=0$. For a cylindrical implosion, in which $\mathbf{v}$ is purely radial and unperturbed quantities are constant over cylindrical surfaces, this can be carried out exactly. Also for the spheromak an initially spherical implosion remains so until near stagnation when the non-uniform pressure of the spheromak causes velocity flows in $\theta$ and $\phi$. Ignoring these velocity components, we integrate Eq. (1) over the liquid layer $\mathrm{R}_{\mathrm{S}}<\mathrm{r}<\mathrm{R}_{\mathrm{o}}$ with $\mathrm{v}_{\mathrm{r}} \propto \mathrm{r}^{-2}$ and solve to obtain the approximate effective gravity at any position on the liquid-plasma interface at $r=R_{S}$ :

$$
\mathrm{g}_{\mathrm{s}}=-\mathrm{dv}_{\mathrm{s}} / \mathrm{dt}=3 / 2\left(\mathrm{v}_{\mathrm{s}}^{2} / \mathrm{R}_{\mathrm{s}}\right)-\left(\mathrm{P}_{\mathrm{s}} / \rho \mathrm{R}_{\mathrm{s}}\right)
$$

where we drop small contributions at the outer surface (thick layer, $R_{S}<R_{o}$ ).

From flux conservation, the magnetic pressure at the liquid surface scales as: 


$$
\mathrm{P}_{\mathrm{S}} \quad=\quad \mathrm{B}^{2} / 2 \mu_{\mathrm{o}}\left(\mathrm{R} / \mathrm{R}_{\mathrm{S}}\right)^{4}
$$

where $\mathrm{B}$ and $\mathrm{R}$ are the field and radius at stagnation $(\mathrm{v}=0)$. Then the solution to Eq. (5) is:

$$
\begin{aligned}
& \mathrm{v}_{\mathrm{S}}=\left[\left(2 \mathrm{P}_{\mathrm{S}} / \rho\right)\left(\mathrm{R}_{\mathrm{S}} / \mathrm{R}-1\right)\right]^{1 / 2} \\
& \mathrm{~g}_{\mathrm{S}}=-\left(\mathrm{P}_{\mathrm{S}} / \rho \mathrm{R}_{\mathrm{S}}\right)\left(4-3 \mathrm{R}_{\mathrm{S}} / \mathrm{R}\right)
\end{aligned}
$$

Instability sets in $(\mathrm{g}<0)$ at $R_{S}=4 / 3 R$, at which point the implosion velocity is maximum:

$$
\mathrm{v} \quad=0.32 \mathrm{v}_{\mathrm{A}}
$$

where $\mathrm{v}_{\mathrm{A}}=\mathrm{B} / \mathcal{}_{\rho} \rho \mu_{\mathrm{o}}$ is the liquid Alfven speed at stagnation. The $\mathrm{g}$ at stagnation is:

$$
\mathrm{g}=-\left(\mathrm{B}^{2} / 2 \mu_{\mathrm{o}}\right) /(\rho \mathrm{R}) \quad=\quad-1 / 2 \mathrm{v}_{\mathrm{A}}^{2} / \mathrm{R}
$$

The effective gravity is largest at the midplane and diminishes toward the geometric axis of the spheromak. The average value is given by energy conservation. From Eq. (6), the magnetic energy inside the spheromak scales as $\mathrm{E}_{\mathrm{M}} \propto \mathrm{R}_{\mathrm{S}}{ }^{-1}$ with derivative:

$$
\mathrm{dE}_{\mathrm{M}} / \mathrm{dt} \quad=\quad-\mathrm{E}_{\mathrm{M}} \mathrm{v}_{\mathrm{S}} / \mathrm{R}_{\mathrm{S}}
$$

while the change in kinetic energy in the liquid layer is:

$$
\mathrm{dE}_{\mathrm{K}} / \mathrm{dt} \quad=\quad\left\langle\mathrm{P}_{\mathrm{S}}>\mathrm{A}_{\mathrm{s}} \mathrm{V}_{\mathrm{s}}\right.
$$

with surface area $A_{S}$. To conserve energy the average pressure on the surface is:

$$
\left.<\mathrm{P}_{\mathrm{S}}\right\rangle=\mathrm{E}_{\mathrm{M}} / \mathrm{A}_{\mathrm{S}} \mathrm{R}_{\mathrm{S}}=1 / 3<\mathrm{B}^{2}>/ 2 \mu_{\mathrm{o}}
$$

with volume average field energy and corresponding $\left\langle\mathrm{g}_{\mathrm{s}}\right\rangle$ by Eq. (8). 


\section{Rotational Stabilization}

For a cylinder, it is possible to suppress Rayleigh-Taylor growth at the interface by rotation that effectively alters $\mathrm{g}$. This requires a rotation speed comparable to the implosion speed so that the required energy is about double that required to compress the plasma [2]. Hence stabilization by rotation reduces the efficiency $\varepsilon$ by a factor of 2 and the gain $G \propto \varepsilon^{2}$ by a factor of 4 [1], thus reducing the gain from $G=20$ to $G=5$ for the cases shown in Reference [1].

Moreover, unlike the cylinder, in the spheromak gravity points in all directions (though progressively weaker in directions along the geometric axis of symmetry) so that rotation about a single axis (the geometric axis) may not be fully stabilizing. Also, whereas the necessary rotation is easily imparted to a cylinder simply by setting the shell in rigid rotation, for the spheromak it would be necessary to tailor the rotational input to spin only the inner surface. The difference lies in the fact that, for the cylinder, both the implosion speed and the injected rotational speed increase as $\mathrm{r}^{-1}$, so that a weak initial rotation matching the implosion speed continues to do so as the implosion proceeds. For the sphere, rotation scales as $r^{-1}$ but the implosion speed scales as $r^{-2}$, so that excess rotation is required initially, tailored to impart no more than is required to stabilize the system at stagnation.

\section{Shear Stabilization}

As is pointed out in Reference [1], the spheromak confined within a rigid flux conserver of spherical or oblate shape can be made stable to ideal MHD modes by virtue of magnetic shear. Thus, by manipulating the injection pressure to insure the proper shape of the liquid boundary, the spheromak can be made internally stable during the compression up to the point that $\mathrm{g}$ reverses sign and Rayleigh-Taylor surface modes develop.

It may be that shear stabilizes or weakens surface modes. For the case of pure growth or oscillation, the shear and gravity effects can be compared through appropriate terms in the perturbed energy:

$$
\delta \mathrm{W} \approx \int \xi^{2}\left(\mathrm{~B}^{2} / 2 \mu_{\mathrm{o}}\right)\left[\left(\rho^{\prime} / \rho \mathrm{R}\right)-\left(1 / \mathrm{RL}_{\mathrm{s}}\right)\right]
$$

where $L_{S}$ is the shear length, $R$ is the radius of the spheromak, and in the first term we introduced the maximum $g \approx-\mathrm{p} / \rho \mathrm{R}$ and $\mathrm{p} \approx \mathrm{B}^{2} / 2 \mu_{\mathrm{o}}$. For a mode of given $\mathrm{k}$, solutions of Eq. (3) are localized within a thickness $\mathrm{k}^{-1}$ around the boundary, where the steep density gradient causes the gravity term to outweigh the shear term even when the sheared field 
diffuses into the liquid. Moreover the magnitude of the maximum gravity overcomes shear even for maximum wavelengths $\mathrm{k} \approx \mathrm{R}^{-1}$ (as can be seen by approximating $\rho^{\prime} / \rho \approx \delta(\mathrm{r}-\mathrm{R}$ ) assuming $\mathrm{R}<\mathrm{L}_{\mathrm{s}}$ ).

\section{Instability Growth Estimates and the Burn Time}

It may be that, because $\mathrm{g}$ is stabilizing ( $>0$ ) until compression is nearly complete, instability does not matter. To see this, we now estimate instability growth and its effect on the burn time.

In Reference [1], we estimated the burn time $\tau=2 \mathrm{R} / \mathrm{v}$ for implosion velocity $\mathrm{v}$, which, in terms of the Alfven speed, was estimated to be $v=v_{A} / \sqrt{ } 3$. The maximum implosion velocity given by Eq. (9) is smaller by a factor $(3 / 4)^{2}$, giving a longer burn time. The actual burn time depends also on the onset of ignition, around $5 \mathrm{KeV}$ in a pure DT plasma. For the examples of Reference [1] based on $\mathrm{T}=10 \mathrm{KeV}$ by compression at stagnation, ignition occurs at $R_{S} / R=\sqrt{ } 2$, giving a shorter $\tau=2(\sqrt{ } 2-1)(R / v) \approx R / v$, which is, however, about the same as the estimate of Reference [1] using instead v in Eq. (9). Thus, given the uncertainties, we conclude that our previous estimate for the burn time is reasonable for a stable implosion, namely:

$$
\tau=2 \sqrt{ } 3 \mathrm{R} / \mathrm{v}_{\mathrm{A}}=3.46 \mathrm{R} / \mathrm{v}_{\mathrm{A}} .
$$

A different estimate would be $\tau=\mathrm{N} \gamma^{-1}$ for some reasonable number of e-folds before instability quenches the burn, $\gamma$ being the instability growth constant.

Though the Rayleigh-Taylor growth rate increases with k, actually only modes of small $\mathrm{k}$ are of interest. The large $\mathrm{k}$ modes grow first and effectively make the pressure gradient at the interface less steep as surface bumps jet inward. By the time the jets protrude a distance comparable to the wavelength, the "mean" state given by the symmetric average is no longer unstable to modes of this wavelength. Progressively the mean surface "diffuses" inward, dominated by longer and longer wavelength distortions -- most importantly those on the order of the radius at which instability sets in. Thus as a "worst case scenario" we consider modes of wavelength $\mathrm{kR}=1$, yielding, by Eqs. (4) and (10):

$$
\gamma \quad=\quad \sqrt{k g} \leq(1 / \sqrt{2}) \mathrm{v}_{\mathrm{A}} / \mathrm{R}
$$


and an instability-limited burn time

$$
\tau \quad \geq \quad \sqrt{ } 2 \mathrm{~N}\left(\mathrm{R} / \mathrm{v}_{\mathrm{A}}\right)
$$

Comparing Eqs. (15) and (17), we see that, even if it occurred, instability would not significantly alter the gain estimated in Reference [1] for a growth period of 2.5 e-folds, giving a perturbation amplification factor $\exp 2.5=12$. Thus growing perturbations would not exceed R (the "encroachment" criterion of Reference [2]) for surface ripples of order $0.1 \mathrm{R}$ (stagnation radius) at the onset of instability. This may not be a very stringent criterion. Actually initial perturbations damp during the stable implosion phase, due to terms omitted in Eq. (3).

\section{Effect on the Gain}

We now revise the estimates of the fusion energy gain $G$ in Reference [1] to take into account the conclusions above.

The gain formula derived in Reference [1] is:

$$
\begin{aligned}
& \mathrm{G}=\delta\left[3.2 \times 10^{-5} \varepsilon^{2} \beta_{\mathrm{o}} \mathrm{R}_{\mathrm{o}} \mathrm{B}_{\mathrm{i}}\left(2 \mathrm{EC} / \mathrm{V}_{\mathrm{o}} \mathrm{B}_{\mathrm{i}}\right)^{1 / 2} / \mathrm{c}_{\mathrm{s}}\right]_{\mathrm{OLD}}\left[\beta_{\mathrm{o}} \mathrm{C}^{1 / 3}\right]_{\mathrm{NEW}} \\
& \varepsilon \quad=\left\{1 / 2\left[1+\left(1+2 \alpha \mathrm{E} \mathrm{C}^{4 / 3} / \mathrm{V}_{\mathrm{o}} \mathrm{B}_{\mathrm{i}}\right)^{1 / 2}\right]\right\}^{-1}
\end{aligned}
$$

where we have added a "NEW" beta factor inadvertently left out in Reference [1] and the factors $\delta=\alpha=1$ in that reference.

To illustrate the new effects, we recalculate $G$ for the old and new formulas for the case $R_{o}=1.77 \mathrm{~m}\left(V_{o}=23.2 \mathrm{~m}^{3}\right)$ and input energy $\mathrm{E}=150 \mathrm{MJ}$ given previously in Table 1 of Reference [1]. Again we assume a LiPb eutectic with bulk modulus $\mathrm{B}_{\mathrm{i}}=2 \times 10^{10} \mathrm{~Pa}$ and sound speed $\mathrm{c}_{\mathrm{s}}=1600 \mathrm{~m} / \mathrm{s}$. The new cases are distinguished by $\alpha=1 / 9$, appropriate if we evaluate the energy of compression, $\int \mathrm{P}^{2} / 2 \mathrm{~B}_{\mathrm{i}}$, using the average pressure in Eq. (13); and also two values of $\delta$, unity and $1 / 3$, representing a burn for 2.5 or 0.8 e-folds, respectively. Results for the gain $\mathrm{G}$ and compression efficiency $\varepsilon$ are given for several values of the volume compression ratio $\mathrm{C}$ : 


$\begin{array}{lccccc}\mathrm{C} & \mathrm{G}_{\mathrm{OLD}} & \varepsilon_{\mathrm{OLD}} & \mathrm{G}_{\mathrm{NEW}}(\delta=1) & \mathrm{G}_{\mathrm{NEW}}(\delta=1 / 3) & \varepsilon_{\mathrm{NEW}} \\ 10 & 6 & 0.99 & 1.2 & 0.4 & 0.99 \\ 100 & 16 & 0.93 & 8.3 & 2.8 & 0.99 \\ 344 & 20 & 0.77 & 22 & 7.3 & 0.96 \\ 1000 & 16.5 & 0.53 & 43 & 14 & 0.86\end{array}$

As we found in Reference [1], for the old formula the maximum gain of 20 corresponds to an optimum efficiency while for the new formula, with less compression energy on average, the optimum occurs at unrealistically large compression ratios.

Once again we find a gain $\mathrm{G} \approx 20$ to be a reasonable estimate consistent with a few e-folds of instability growth.

\section{Conclusions}

The conclusion from Reference [1] that ignition of a spheromak by compression appears feasible with fusion energy gains $\approx 20$ is reconfirmed, even taking into account the potential for Rayleigh-Taylor instability. An imploding thick layer of conducting liquid can provide a stable container for a spheromak throughout spherical compression, until the spheromak radius is $3 / 4$ that at stagnation, and the volume compression ratio is $40 \%$ of its final value. At this time, by virtue of spherical convergence, the implosion velocity is 1000 times its initial value, so that very slow initial compression -- over a fraction of a second -can lead to strong compression at stagnation. By letting the slowly moving liquid layer implode freely, the kilobar pressures attained at ignition are absorbed by the liquid rather than the wall.

Thus it appears that liquid evaporation and transport into the plasma, not stability, is the main area of physics on which the ultimate feasibility of the concept depends.

\section{References}

[1] T. K. Fowler, "Pulsed Spheromak Reactor with Adiabatic Compression," Lawrence Livermore National Laboratory Report, UCRL-ID-133884, April 23, 1999.

[2] A. Barcilon, D. L. Book and A. L. Cooper, "Hydrodynamic stability of a rotating liner," Physics of Fluids, 17, 1707 (1974).

[3] T. Dolan, Fusion Research, Pergamon Press, Oxford, 1982, Chapter 8. 